\title{
IMPACTO DE LAS REDES SOCIALES SOBRE EL DEBATE POLÍTICO ${ }^{1}$
}

\section{IMPACT OF SOCIAL MEDIA IN POLITICAL DEBATE}

\author{
Gabriel Alconchel \\ Universidad Nacional de Educación a Distancia (UNED) \\ galconche1@alumno.uned.es
}

\begin{abstract}
Resumen: Este artículo analiza el impacto de las redes sociales sobre el debate político, a partir del análisis del debate sobre el estado de la nación de 2013 en España. Se definen los contenidos predominantes en las conversaciones que se mantienen en Twitter durante la semana en la que se desarrolla el debate: del lunes 18 de febrero al domingo 24 de febrero, y se compara el grado de interés de los usuarios en relación con otros asuntos. A continuación, se examina la dimensión y el alcance del debate en esta red social. Se analiza la valoración de los usuarios sobre el Gobierno, las reacciones ante el diagnóstico del presidente del Gobierno sobre la situación del país y el sentido de los mensajes de los usuarios respecto al líder de la oposición. También se explora el tono de los mensajes y se comprueba si la conversación sobre el debate en Twitter gira en torno a los mismos temas y problemas que los ciudadanos consideran más relevantes según los datos que ofrece el Centro de Investigaciones Sociológicas. Los resultados contribuyen a comprender más adecuadamente el impacto de Twitter en la configuración de la opinión pública.
\end{abstract}

Palabras clave: Internet, Twitter, redes sociales, política, esfera pública, cultura política.

\begin{abstract}
Based on an analysis of the debate on the state of the nation in 2013 in Spain, this article analyzes the impact of social networks on political debate. The predominant content in conversations on Twitter during the week in which the debate takes place (Monday 18 February to Sunday 24 February) are defined, and the degree of interest of users with regard to other matters is compared. Then, the size and scope of the discussion in this social network is examined. User ratings of the government are analyzed, as are reactions to the evaluation of the prime minister on the situation and the meaning of user messages to the opposition leader. The study also explores the tone of the messages and checks to see if the debate on Twitter revolves around the same issues and problems that citizens consider most relevant according to data provided by the Center for Sociological Research. The results contribute to a better understanding of the impact of Twitter on public opinion.
\end{abstract}

Key words: Internet, Social media, Twitter, Political parties, public sphere, Political Culture.

1 Los contenidos de esta publicación son parte de los trabajos de preparación de la tesis doctoral del autor en la Universidad Nacional de Educación a Distancia (UNED). 


\section{Introducción}

Las funciones y procesos dominantes en la era de la información cada vez se organizan más en torno a redes. Y esta nueva morfología social y la difusión de su lógica modifican la operación y los resultados de los procesos de producción, la experiencia, el poder y la cultura (Castells, 1998a). En el origen de esta investigación está el debate sobre si la comunicación on line a través de las redes sociales es un instrumento de contestación social y política, o si, por el contrario, se trata de un artilugio orientado mayoritariamente a contenidos funcionales y de legitimación del orden social y político.

La investigación se focaliza en el impacto de la red social Twitter en los procesos de comunicación y sus potencialidades desde el punto de vista político. Para ello, se elige un acontecimiento político relevante desde el punto de vista institucional en España: el debate sobre el estado de la nación que se celebra con carácter anual en el Congreso de los Diputados, la principal cámara legislativa, y en el que el presidente del Gobierno de manera global debe dar cuenta en el parlamento de su gestión ante las fuerzas políticas de la oposición. El 16 de febrero de 2013, el Congreso de los Diputados de España, a la vez que informaba sobre la fecha de celebración del debate en una nota de prensa ${ }^{2}$, lanzaba el hashtag \#DEN2013, para que los tuiteros comentaran el debate sobre el estado de la nación en torno a esa etiqueta. Este trabajo analiza la conversación que se produce en España en la red social Twitter sobre el debate sobre el estado de la nación.

\section{Enfoque teórico}

Las comunidades en las redes sociales no son un elemento abstracto independiente de la realidad social. Pero, no cabe duda que la comunicación en las redes sociales fabrica identidades sociales y nuevos escenarios y espacios públicos. En los actos comunicativos en Internet van implícitos los esquemas culturales asimilados por sus usuarios, por lo que el grado de libertad que se pueda ejercer en la red estará vinculado a la condición social y económica en el mundo real (Contreras, 2009).

Con las revueltas políticas en el Norte de África y Oriente Medio de 2011, Twitter adquirió una nueva dimensión (Ben Jeloun, 2011). Se abrió la veda para todo tipo de relaciones entre tecnología y sociedad. Los amantes de las TIC vieron la oportunidad perfecta para proclamar a la red como la gran catalizadora y disparadora de los cambios sociales.

En este trabajo, ni se abraza un reduccionismo tecnológico que proclama las bondades de todo lo tecnológico como nuevo tótem, ni se valora cualquier innovación tecnológica como una patraña superestructural promovida por el anarcocapitalismo de nuevo cuño que aspira a nuevos mercados, nuevas ganancias y a una nueva forma de organización social (Ippolita, 2012). Este trabajo de investigación analiza el fenómeno de las redes sociales desde un punto de vista crítico para aproximarse a los modelos actuales de comunicación y a una crítica de la democracia representati-

2 Nota de prensa del Congreso informando sobre la celebración del debate sobre el estado de la nación. Disponible en: http://www.congreso.es/portal/page/portal/Congreso/Congreso/SalaPrensa/NotPre?_piref73_7706063_73_1337373_1337373.next_page=/wc/detalleNotaSalaPr ensa\&idNotaSalaPrensa $=8863 \&$ anyo $=2013 \&$ mes $=2 \&$ pagina $=1 \&$ mostrarvolver $=S \&$ movil=nul [16-02-2013]. 
va vigente en los países occidentales. A partir de ahí, realiza un análisis que permite conocer de manera objetivable aspectos concretos de un acontecimiento político relevante, para, a continuación, inferir conclusiones de este análisis que hagan posible abrir futuras líneas de investigación que permitan analizar la comunicación en el ciberespacio y el alcance de la ciberpolítica.

Utilizando a Habermas (2010) y su concepto procedimental de democracia, gracias a las TIC sería posible introducir mejoras en los procedimientos para el debate político y la toma de decisiones. A este concepto se le unen reflexiones como las de Thompson (1998), que insiste en la necesidad de encontrar maneras de incrementar la participación democrática mediante la ampliación de los procesos deliberativos. Es decir, afirma que es posible resolver cuestiones de forma creativa y colaborativa en red.

En el análisis del acto comunicativo que tiene lugar en las redes sociales la interactividad ha generado un verdadero campo de batalla sobre las posibilidades del diálogo y el alcance en los nuevos soportes técnicos.

En este debate es posible encontrar desde el paradigma infomacional de Castells (1998a), hasta la apología del hombre como sujeto que va perdiendo capacidad de análisis racional, crítica y, sobre todo, capacidad de abstracción para aprehender, interpretar y dotar de sentido el mundo que le rodea y a sí mismo (Sartori, 1998). Es decir, una suerte de vuelta a la caverna como poéticamente profetizaba Saramago.

En esta investigación, se parte de la idea que la tecnología no determina la sociedad, pero tampoco la sociedad determina exclusivamente la innovación tecnológica (Castells, 1998a). Esta dialéctica entre sociedad y tecnología hunde sus raíces en cómo se configuran las relaciones económicas y en cómo interactúan con las pautas culturales.

Esta investigación no abraza la dimensión exclusivamente técnica del paradigma tecnocientífico. Pero las funciones y procesos dominantes en la era de la información cada vez se organizan más en torno a redes. Y esta nueva morfología social y la difusión de su lógica modifica la operación y los resultados "de los procesos de producción, la experiencia, el poder y la cultura” (Castells, 1998a: 505).

Estos cambios provocan transformaciones en el proceso político democrático de las sociedades capitalistas occidentales, que generan desafíos pero también oportunidades. En este sentido, las redes sociales permiten configurar mecanismos para una política encaminada a procesar de manera inmediata valores y opiniones públicas. Lo que abre nuevas expectativas a la reorganización de las relaciones de poder en las sociedades democráticas.

\section{Objetivos}

Twitter es una plataforma en línea para publicar y leer mensajes de texto de hasta 140 caracteres. Fue creada en 2006 por Jack Dorsey, Biz Stone y Evan Williams. Comenzó siendo un desarrollo interno de la compañía Odeo para agilizar las comunicaciones entre sus empleados. En junio de 2006 se abrió al público y desde entonces no ha dejado de crecer.

La liberación de la Interfaz de Programación de Aplicaciones (API) de Twitter ha permitido la creación de miles de desarrolladores que han mejorado sus funciones, simplificando técnicamente una API es una biblioteca de código que permite ser utili- 
zada por otro software. La apertura del código de Twitter es parte del éxito de la red social junto a su modelo comunicativo.

Parece muy sencillo explicar qué es Twitter. Es una herramienta de microblogging que permite enviar mensajes de 140 caracteres y seguir a las personas que se consideren interesantes. Sin embargo, resulta más complicado desentrañar todo lo que rodea a esta red social. Y es que "Twitter ha cambiado la red y ha completado el giro social que iniciaron los blogs a finales de los años noventa" (Orihuela, 2011).

La información en Twitter fluye de manera viral a partir de la creación de un mensaje y su posterior redifusión por la red de influencia de la persona que lo emite y de las redes de los que lo reciben y deciden volver a compartirlo, y así sucesivamente.

Su fuerza radica en parte por el uso de etiquetas que hacen que vuelen las noticias y las conversaciones (Gómez Díaz, 2012). Y su relevancia para la comunicación política está fuera de toda duda (Gutiérrez-Rubí, 2011). Políticos y gobiernos a través de esta red pueden compartir, conversar, escuchar, reducir el tiempo de espera y crear una relación más directa con el ciudadano. Y también a la inversa. Para los ciudadanos y los movimientos sociales la red les ayuda a compartir, conversar, escuchar, crear una relación directa con los participantes, coordinarse en el movimiento y programar acciones. Es decir, un instrumento de comunicación política sencillo, accesible y barato.

Lo cierto es que Twitter se ha convertido en una de las redes más influyentes a la hora de configurar la opinión pública a escala mundial de manera inmediata e instantánea. Millones de usuarios en todo el planeta confirman a este microblogging como una plataforma con una notable influencia social y política.

Los objetivos de la investigación se centran, en primer lugar, en conocer si los temas del momento o trending topics (TT) en Twitter responden mayoritariamente a asuntos que no tienen que ver con la política. En segundo lugar, se quiere conocer si los usuarios de Twitter utilizaron mayoritariamente esta red social durante el debate sobre el estado de la nación para criticar al Gobierno. Para ello se analiza si los tuits sobre el debate contienen un número elevado de insultos, si los tuits emitidos durante el debate critican más al presidente del Gobierno que al líder de la oposición y, por último, si los principales problemas para los españoles, según el Centro de Investigaciones Sociológicas (CIS), están presentes en los mensajes sobre el debate.

\section{Metodología}

Se aplica la metodología cualitativa para desentrañar los contenidos de las conversaciones en Twitter. Se utiliza el análisis de contenido como metodología principal. Esto va a permitir descubrir y analizar el contenido de las conversaciones en Twitter y el sentido de las mismas en lo referido al debate sobre el estado de la nación.

El estudio se centra en dos fases. En la primera, se averigua a través de un análisis de contenido temático los tipos de conversaciones en Twitter. Con ello se pretende saber de qué se habla en Twitter. Se limita el ámbito de trabajo a la lista de temas del momento que ofrece la red social. Se analiza el listado de temas del momento para el ámbito geográfico de España durante la semana en la que se desarrolla el debate sobre el estado de la nación: entre el lunes 18 de febrero y el domingo 24 de febrero de 2013. 
El listado de los 10 temas del momento en España va cambiando constantemente, debido al carácter instantáneo de los mensajes en Twitter. Para ello, se opta por recoger una muestra representativa de las palabras o etiquetas que son trending topic. Además, se elabora una ficha de análisis más completa que sirve para contextualizar el tema al que se refiere el trending topic. La unidad de registro (Bardin, 1986), es decir, la unidad de significación que vamos a codificar, será el trending topic. Y la unidad de contexto, que permite la comprensión de la unidad de registro, será el tuit más retuiteado ${ }^{3}$ del trending topic.

Blanco Leal (2008) ofrece un modelo de análisis aplicado a los diarios de información general que ha servido de inspiración y base para elaborar un nuevo un sistema de categorías. A partir de ahí, se extrajeron unas categorías comunes. Se definieron y se validaron de manera satisfactoria: política, economía, sociedad, comunicación, cultura y deportes. Con ello, se intenta reducir la dificultad de la dispersión y la complejidad. Pero todavía quedaba el asunto de las cuestiones cotidianas que los usuarios comentaban en Twitter como "Buenos días" o "Feliz martes" y que se convertían en trending topic.

Revisando la tradición del análisis de contenido, se utilizó una de sus reglas: la de adecuación o pertinencia. Es decir, adaptar las categorías al objeto de estudio. En virtud de esta regla se incluyó la categoría de tuiterías. Así, se define esta categoría como las actualizaciones banales que en ocasiones publican los usuarios de Twitter para anunciar actividades de su vida cotidiana.

Para la recogida de esta información y la realización del análisis temático se elabora un sistema de categorías y se realiza una ficha de análisis que permite codificar y sistematizar la información para tratarla de manera agregada.



Gráfico 1. Ficha de registro de los temas del momento. Fuente: Elaboración propia.

La recogida de datos se realiza tres veces al día. A las 9,00 horas, a las 16,30 horas y a las 22,00 horas. A diferentes horas, para mitigar la influencia de los contenidos que se emiten desde los medios de comunicación tradicionales en el listado de trending topic. Ya que el listado de tendencias suele estar muy influido por el prime time televisivo de cada noche. Siempre, la recogida de datos se efectúa a las mismas horas cada día durante la semana del 18 al 24 de febrero. En total, se obtienen 30 trending topics diarios, lo que supone 210 trending topics durante la semana. Una muestra

3 Es una información que nos ofrece Twitter al hacer clic en el trending topic que elijamos. 
significativa para extraer conclusiones. Además, se partía de la premisa de eliminar, si los hubiera, los trending topics patrocinados. Aunque en la muestra de 210 temas del momento no hubo finalmente ningún trending topic ni tuit patrocinado.

Pero, aunque los trending topics son una buena manera de conocer en torno a qué asuntos giran los debates virtuales, debe tenerse en cuenta que no siempre son precisos a la hora de valorar el impacto de un tema en Twitter. La lectura en Twitter (Polo, 2012) se produce de diferentes modos. En primer lugar, se interactúa sobre lo que aparece en el timeline del usuario, que es lo que escriben los demás usuarios a los que sigue. En segundo lugar, hay que tener en cuenta que Twitter ofrece la posibilidad de clasificar las cuentas a las que se sigue por listas en función de ámbitos de relación o asuntos de interés. Por lo que accediendo a listas se puede interactuar con tuiteros afines a algún tema. Además, las conversaciones en Twitter giran en torno a etiquetas. $Y$ puede haber debates muy relevantes que se siguen de forma sencilla sin necesidad de que sean trending topics.

Además, hay que tener en cuenta que Twitter utiliza un algoritmo secreto para clasificar los temas del momento. Y no siempre coincide el resultado de este algoritmo con el interés de los usuarios, ni desde un punto de vista cualitativo, ni tampoco cuantitativo. De este modo, que una palabra o etiqueta sea trending topic no quiere decir que sea el asunto sobre al que más tuits se envían.

Por eso, se ha planificado una segunda fase en la metodología, que se adecua más a la manera en que se conversa en Twitter. En la segunda fase, se realiza un análisis de los tuits que los usuarios emiten acerca del debate sobre el estado de la nación. En este caso, los tuits que se emiten con la etiqueta que se utiliza mayoritariamente para el debate: el hashtag \#DEN2013. Esta fase de la investigación se centra en averiguar, por una parte, si el sentido de los mensajes es favorable o contrario al Gobierno y cómo se valora a los dos principales líderes políticos. Y, por otra, en conocer el tono de los mensajes y si estos se refieren a los asuntos que los españoles señalan como los principales problemas en los sondeos que elabora el Centro de Investigaciones Sociológicas. Según el CIS, estos asuntos eran en el momento de la celebración del debate sobre el estado de la nación: el paro $(79,8 \%)$, la corrupción $(40 \%)$ y la situación económica $(35,3 \%)^{4}$.

Para esta fase de la investigación se utiliza el análisis de contenido semántico para conocer los significados de los tuits, yendo más allá de la literalidad de las palabras que lo componen, y analizar el significado político de los mismos. El análisis de contenido "es sensible al contexto, y por lo tanto, es capaz de procesar formas simbólicas" (Krippendorff, 1990: 28). Para el análisis se limita la selección del universo a los tuits emitidos durante la parte más seguida y comentada del debate. Es decir, los tuits que se emiten durante el "cara a cara" entre el presidente del Gobierno y el líder de la oposición parlamentaria.

El número total de tuits emitidos con el hashtag \#DEN2013 durante el 20 y el 21 de febrero fueron 201.589. El cara a cara entre Rajoy y Rubalcaba tuvo lugar el 20 de febrero de 2013 entre las 16,00 horas y las 17,52 horas. Para el análisis, se examinan los tuits que se emiten sobre el debate entre las 16,00 horas y las 18,00 horas del 20 de febrero. En total se emitieron durante este periodo de tiempo con el hashtag \#DEN2013 un total de 28.783 tuits. El uso de la muestra facilita la codificación en los términos pretendidos en esta investigación. Un procedimiento por el que se infieren los

4 Barómetro de febrero 2013 del CIS. Recuperado de http://www.cis.es/cis/export/sites/default/Archivos/Marginales/2960_2979/2978/Es2978.pdf. 
valores verdaderos de una población a través de la experiencia obtenida con un grupo que contiene un número menor de casos que la población (García Ferrando, 1996).

Para determinar el tamaño de la muestra se sigue a García Ferrando (1996). Así, a pesar de que se podría haber elegido un nivel del $95 \%$, se decide elegir un nivel de confianza del $99 \%$ y un error muestral de $\pm 3 \%$. La elección de un mayor nivel de confianza ofrece mayores garantías de que la estimación efectuada a través de la muestra se aproxime más al valor real del parámetro. De este modo, la muestra -en función a estos criterios estadísticos- serán los 1.737 tuits elegidos al azar, a través de un muestreo aleatorio simple, entre los 28.738 que forman la población de referencia. Con ello se obtienen los tuits que se analizan de manera pormenorizada y sobre los que se efectúa el análisis de las variables que permitan confirmar o desestimar la hipótesis, determinar el cumplimiento de los objetivos y emitir unas conclusiones finales. El análisis sobre el sentido de los tuits se realiza a partir de una serie de variables que están relacionadas con los objetivos de esta parte del análisis. Para el tratamiento de la información se utiliza el programa informático de procesamiento de datos SPSS.

\begin{tabular}{|llll|}
\hline \multicolumn{2}{|c|}{ Variables } \\
Valoración de líderes & Valoración del Gobierno & \multicolumn{1}{c|}{ Tono del tuit } & Tema del tuit \\
0 Ninguna & & & \\
1 Positiva Rajoy & 0 Neutra & 0 Otros & 0 Otros temas \\
2 Negativa Rajoy & 1 Positiva & 1 Positivo & 1 Paro \\
3 Positiva Rubalcaba & 2 Negativa & 2 Crítico & 2 Corrupción \\
4 Negativa Rubalcaba & & 3 Informativo & 3 Crisis Económica \\
5 Negativa Rajoy y Rubalcaba & & 4 Humorístico & \\
\hline
\end{tabular}

Gráfico 2. Variables para clasificar los tuits con la etiqueta \#DEN2013.

Fuente: Elaboración propia.

\section{Resultados}

Los temas que predominan en el listado de temas del momento son variados. La primera área temática es la de Comunicación (24\%), aunque seguida por los temas de Política (23\%). Además, de los clasificados como Comunicación, el $75 \%$ estaban relacionados con comentarios sobre programas de televisión y radio. Este tipo de TT supone el $18 \%$ del total de tendencias.

\section{Análisis temático trending topics}



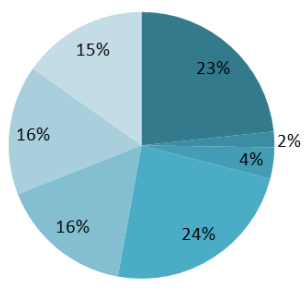

Gráfico 3. Análisis temático de los trending topics. Fuente: Elaboración propia. 
A pesar de ello, como se ha señalado, el $23 \%$ de los temas del momento durante la semana del debate sí tuvieron que ver con la política y el 7\% estaban directamente relacionados con el debate.

De los trending topics sobre temática política, el $48 \%$ tiene un tono informativo, el $44 \%$ tienen un tono crítico y el $6 \%$ tienen un carácter positivo. Sólo un tuit de los más retuiteados que provocan los trending topics sobre política está redactado en tono de humor. De los trending topics de la temática Deportes, el $81 \%$ se refieren a fútbol. Del total de trending topics sobre fútbol, el $96 \%$ fueron tendencia durante el fin de semana.

El $91 \%$ de los retuits en tono de humor son emitidos por usuarios ficticios. Ninguno de los tuits más retuiteados que provocan los trending topics sobre tuiterías están emitidos por usuarios identificables como personas físicas. El tono más utilizado es el tono positivo (48\%). Le siguen los tuits en tono informativo (19\%). El porcentaje de tuits con tono humorístico superan a los de tono crítico.

Si se compara el tono de los tuits en el análisis de los trending topics con el del análisis de los tuits con la etiqueta \#DEN2013, se concluye que el debate político en Twitter tiene sus propias particularidades, ya que el humor no es tan valorado como en otro tipo de conversaciones.

Respecto a la segunda fases del análisis, hay un mayor número de tuits que se refieren a Mariano Rajoy. Si se eliminan los tuits que no hacen referencia a ninguno de los dos líderes, 1.443 tuits emiten una valoración de Rajoy y Rubalcaba, ya sea en sentido positivo o en sentido negativo. El $44,5 \%$ de los tuits están referidos a Mariano Rajoy. El 17,9\% muestran una valoración positiva del presidente del Gobierno y el $26,6 \%$ se muestran contrarios a su figura. El $27,8 \%$ de los tuits se refieren al líder del principal partido de la posición. De los tuits que versan sobre Rubalcaba, un 17,3\% muestra una valoración positiva y un $10,5 \%$ lo citan de un modo negativo.

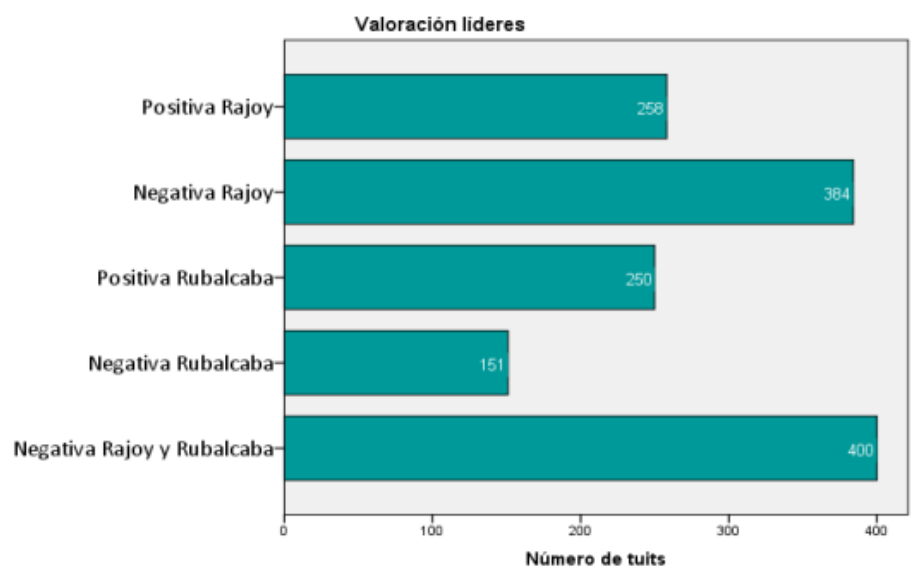

Gráfico 4. Valoración de líderes. Fuente: Elaboración propia.

El mayor porcentaje de tuits se refieren negativamente a los dos líderes políticos, un $27,7 \%$ de los tuits. En términos absolutos, 1.055 tuits muestran una valoración negativa del Gobierno. 288 se expresan a favor del Gobierno. Y 394 se manifiestan de manera neutra. El 60,75\% expresaron una opinión negativa del Gobierno. Un 16,6\% hace una valoración positiva del Gobierno. Atendiendo al clima político y a la situa- 
ción económica, hay que destacar que es un porcentaje considerable. A pesar que Mariano Rajoy tiene una valoración mayoritariamente negativa, esta es menor que la valoración negativa global que tienen del Gobierno. Rajoy no despierta un fuerte rechazo en los usuarios de Twitter.

Los tuits críticos con el presidente del Gobierno son el $26 \%$. Los que critican a Rubalcaba son el 10,5\%. Hay que tener en cuenta del menor nivel de interés que despierta el líder de la oposición en función de los tuits analizados, además de que es el presidente del Gobierno quien se examina de su gestión en un debate como el del estado de la nación.

Respecto al apoyo a ambos líderes, el porcentaje de tuits es parecido, 17,9\% y $17,3 \%$, respectivamente. Hay coincidencia entre la valoración sobre el presidente del Gobierno en el sondeo del CIS sobre el resultado del debate y la valoración de los tuiteros. Mientras que el $26 \%$ de los ciudadanos valoraron negativamente a Mariano Rajoy durante el debate, la valoración negativa de los tuiteros alcanzó el 27\%.

El $77,35 \%$ de los tuits emitidos se ajustan a la dinámica del debate parlamentario: utilizan Twitter para expresar su opinión sobre el Gobierno de un modo abierto. Respecto al tono del tuit, destaca el tono crítico de más de la mitad de los tuits emitidos. Aunque también hay un importante número de tuits con un tono positivo: un $29,4 \%$. Los tuits que están redactados en tono de humor alcanzan los 115, lo que supone un $6,6 \%$ del total de los tuits emitidos.

Los tuits que contienen insultos son 105 , un $6 \%$ del total. Se confirma que los tuits sobre el debate no contienen un número elevado de insultos, a pesar del clima de opinión en el país. De los 1.737 tuits analizados, sólo 105 tuits contenían insultos. En la tarea de análisis de los tuits y codificación a través del programa SPSS se detecta que los insultos no provenían mayoritariamente de usuarios con avatares y biografías ficticias, sino que procedían aparentemente de personas reales. No se utilizan las posibilidades que ofrece Twitter de esconderse tras un perfil ficticio para emitir insultos durante el debate.

Los principales problemas de los ciudadanos, según el CIS, no se reflejan en el debate en Twitter. Los tuits que giraron en torno al principal problema del país -según los resultados del CIS- el paro, fueron 83, y ello a pesar de los casi seis millones de parados.

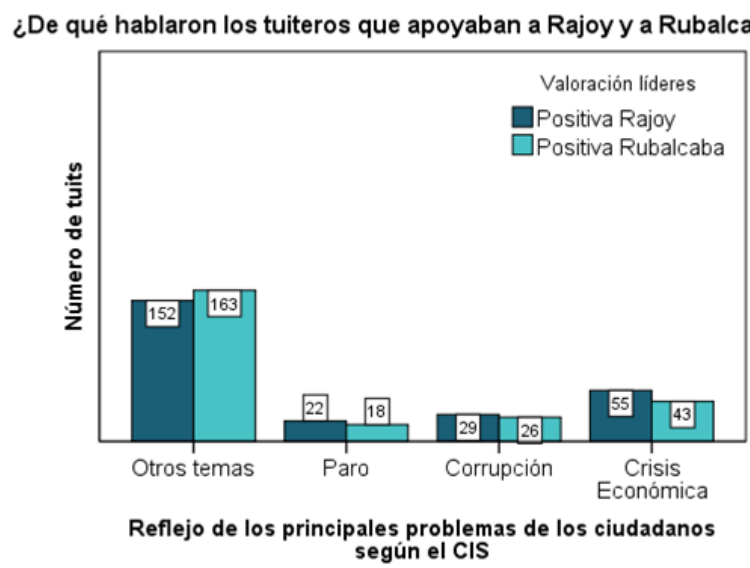

Gráfico 5. Los principales problemas según el CIS y su reflejo en los tuits con la etiqueta \#DEN2013. Fuente: Elaboración propia. 
El $74 \%$ de los tuits no se referían a estos temas -dentro este porcentaje destacan los tuits que trataban sobre vivienda-. Frente a los tuits que tratan sobre otros asuntos, la representatividad de los tres problemas que los ciudadanos expresan como los más importantes a través del sondeo del CIS es la siguiente: $11 \%$, corrupción; $10 \%$, crisis económica y $5 \%$, paro. Muchos comentarios se centraron en aspectos colaterales del debate.

\section{Conclusiones}

El anonimato en Twitter no desemboca en insultos que anulan el debate político en la red. En los trending topics analizados no se ha encontrado ningún insulto. Respecto al análisis de los 1.737 tuits con el hashtag \#DEN2013 se han encontrado 105 tuits con insultos, lo que supone un $6 \%$. Los usuarios que hablan sobre política son mayoritariamente personas físicas en función de su biografía, su avatar y sus últimos tuits.

En el debate político en Twitter no hay sólo hooligans. El $65 \%$ de los tuits no apoyan ni a Rajoy ni a Rubalcaba, lo que a priori hace pensar que el grueso de los usuarios no son militantes del PP ni del PSOE. Sobre todo, si tenemos en cuenta que en las últimas elecciones generales entre los dos partidos aglutinaron el 73,35\% de los votos y, en el barómetro del CIS de febrero de 2013 , el $54,7 \%$ de los encuestados manifestaron haber votado a estas dos fuerzas políticas. Esto no quiere decir que no haya presencia de los partidos en las conversaciones en Twitter sobre el debate del estado de la nación. De hecho, el triunfo de etiquetas como \#LasMedidasdeRajoy propuestas por los partidos responden a una movilización de sus militantes y simpatizantes.

Las conversaciones sobre el \#DEN2013 no rebasan ni socavan la democracia representativa. El $83 \%$ de los tuits analizados en el "cara a cara" opinaba de una manera coherente sobre Rajoy y Rubalcaba. Debaten sobre lo que se les propone desde las instituciones políticas y los medios de comunicación tradicionales. Los inconvenientes del anonimato son superados por los beneficios de la instantaneidad y de las posibilidades de diálogo que ofrece. No hubo una contestación radical al debate a través de Twitter. Se respetaron las reglas del juego. El uso de las TIC perfecciona la democracia representativa, abriendo nuevos cauces de comunicación entre representantes y representados más allá de las vías institucionales.

Twitter no es sinónimo de activismo. Twitter no es un instrumento crítico por naturaleza. El $48 \%$ de los tuits más retuiteados que provocan los trending topics poseen un tono positivo. Y el humor casi iguala a la crítica. De los trending topics sobre temática política, el $48 \%$ tiene un tono informativo, el $44 \%$ tienen un tono crítico y sólo el $6 \%$ tiene un carácter positivo. Aunque también es cierto que, en momentos concretos y sobre temas determinados, Twitter se convierte en una red que favorece la generación de conversaciones colectivas que pueden suponer un freno a determinados abusos del poder político y económico. Si analizamos el tono de los tuits más retuiteados que propician los TT que tratan sobre asuntos políticos, se invierte la balanza y la crítica supera a los tuits de apoyo o asentimiento ante un tema. Además, hay que insistir en que las conversaciones en Twitter no equivalen a la opinión pública, pero sí es un ámbito donde se expresan los líderes de opinión de un determinado ámbito. De este modo, los usuarios de Twitter no es que se vuelvan activistas, lo que hacen es confirmar lo que leen en su timeline a través de los retuits que realizan de los mensajes de los líderes de opinión con los que simpatizan. 
Twitter no es el territorio de los prosumers. Los resultados de la investigación muestran las redes sociales como una herramienta de democratización de la información que transforma a las personas en receptores y en productores de contenidos. Pero la función de prosumer ${ }^{5}$ en la actualidad queda reservada a una parte de los usuarios, a los líderes de opinión de cada ámbito. Estos datos ponen en cuestión a Twitter, las redes sociales y la cultura 2.0 como el territorio donde los usuarios consumen, pero también producen información. En este sentido, es obligado rechazar la definición de redes sociales como una herramienta de democratización de la información que transforma a las personas en receptores y en productores de contenidos. Aunque el cambio significativo es que hay más líderes de opinión accesibles al gran público que en ningún momento de la historia contemporánea.

De los 1.737 tuits analizados, el $48 \%$ eran retuits. Si analizamos el universo, la proporción de retuits es equivalente: 14.576 retuits de 28.783 mensajes, es decir, un $50 \%$. En sintonía con el $48 \%$ de retuits que aparecen en la muestra. Por tanto, alrededor de la mitad de los tuits analizados no eran mensajes originales, si no que la acción de los usuarios se limitaba a una actitud pasiva. Aunque no tan pasiva, como la de no participar de ninguna manera en el debate. Se da una apariencia de participación o una apariencia de elaboración de una opinión, más que una reflexión compleja.

\section{Bibliografía}

Azorín, D. (2012). Análisis cibermétrico de los contenidos de Twitter en España 2011. Universidad Politécnica de Valencia. Recuperado de http://riunet.upv.es/bitstream/ handle/10251/17326/Memoria.pdf?sequence=1.

Bardin, L. (1986). El análisis de contenido. Madrid: Akal.

Ben Jelloun, T. (2011). La primavera árabe. El despertar de la dignidad. Madrid: Alianza Editorial.

Berelson, B. (1952). Content Analysis in Communications Research. Nueva York: Hafner Press.

Berger, P. y Luckmann, T. (1991). La construcción social de la realidad. Buenos Aires: Amorrortu.

Blanco Leal, M. (2008). Modelos de Análisis para el estudio crítico de la prensa. Madrid: Ediciones Internacionales Universitarias.

Castells, M. (1998a). La era de la información. Economía, Sociedad y Cultura. Vol. 1. La sociedad red. Madrid: Alianza Editorial.

Castells, M. (1998b). La era de la información. Economía, Sociedad y Cultura. Vol. 2. El poder de la identidad. Madrid: Alianza Editorial.

Castells, M. (2012). Redes de indignación y esperanza. Madrid: Alianza Editorial.

Centro de Investigaciones Sociológicas (2013). Avance de resultados. Debate sobre el estado de la nación (XXIII). Recuperado de http://datos.cis.es/pdf/Es2980mar_A.pdf.

5 La palabra prosumidor, o también conocida como prosumer, es un acrónimo formado por la fusión original de las palabras en inglés producer (productor) y consumer (consumidor). Fue divulgada por el autor Alvin Toffler en La tercera ola. 
Centro de Investigaciones Sociológicas (2013). Barómetro de febrero 2013. Recuperado de http://www.cis.es/cis/export/sites/default/-Archivos/Marginales/2960_2979/2978/ Es2978.pdf.

Contreras, F. (2009). Re(d)unidos: cultura, innovación y comunicación. Barcelona: Antropos.

Eco, U. (2011). Apocalípticos e integrados. Barcelona: Debolsillo.

De Vreese, C. (2007). "Digital renaissance: young consumer and citizen?". En The Annals of the American Academy of Political and Social Science, $\mathrm{n}^{\circ}$ 611, 207-216. DOI: $10.1177 / 0002716206298521$.

Díaz Moreno, V. (2012). "Desigualdades generacionales y nuevos movimientos sociales". En José Félix Tezanos (ed.), Los nuevos problemas sociales: Duodécimo Foro sobre tendencias sociales (353-373). Madrid: Sistema.

García Ferrando, M.; Ibáñez, J. y Alvira, F. (1996). El análisis de la realidad social. Métodos y técnicas de investigación social. Madrid: Alianza Editorial.

Go, A.; Bhayani, Richa y Huang, L. (2009). Twitter sentiment analyisis. CS224N Final Project Report. Stanford University. Recuperado de http://s3.eddieoz.com/docs/sentiment_analysis/Twitter_Sentiment_Classification_using_Distant_Supervision.pdf.

Gómez-Díaz, R. (2012). Etiquetar en la web social. Barcelona: Editorial UOC.

Guadián Orta, C.; Rangel Pardo, F. y Linares Salas, J. (2012). Análisis de Redes de Influencia en Twitter. II Congreso Español de Recuperación de Información. Valencia, junio de 2012. Recuperado de http://users.dsic.upv.es/grupos/nle/ceri/papers/ ceri2012_guardian.pdf.

Gutiérrez-Rubí, A. (2011, 4 de julio). "Twitter, mucho más que la CNN”. El Periódico de Catalunya. Recuperado de http://www.elperiodico.com/es/noticias/opinion/ twitter-mucho-mas-que-cnn-1064457.

Habermas, J. (1966). Teoría y Práctica: ensayos de filosofía social. Buenos Aires: Sur.

Habermas, J. (2010). Facticidad y validez. Sobre el Derecho y el Estado democrático de derecho en términos de teoría del discurso. Madrid: Trotta.

Ippolitia A.C. (2012). En el acuario de Facebook. El resistible ascenso del anarco-capitalismo. Madrid: Enclave de libros.

Joyanes, L. (1997). Cibersociedad. Los retos sociales ante un nuevo mundo digital. Madrid: McGraw-Hill.

Kripendorff, K. (1990). Metodología de análisis de contenido: teoría y práctica. Barcelona: Paidós.

Orihuela, J. L. (2011). Mundo Twitter. Madrid: Alienta.

Pasquali, A. (1972). Comunicación y cultura de masas. Caracas: Monte Ávila.

Pérez Latre, F. (2010): “Las nuevas redes sociales: ¿moda o revolución?” Nuestro Tiempo, enero/febrero, 52-61.

Pérez Tapias, J. A. (2003). Internautas y náufragos. La búsqueda del sentido en la cultura digital. Madrid: Trotta.

Polo, J. y Polo, J.L. (2012). \#Socialholic. Todo lo que necesitas saber sobre el marketing en medios sociales. Barcelona: Gestión 2000. 
Robles, M.; Molina, O. y De Marco, S. (2012). "Participación política digital y brecha digital política en España. Un estudio de las desigualdades digitales". Arbor. Ciencia, Pensamiento y Cultura, n 188, julio-agosto, 756. DOI: 10.3989/arbor.2012.756n4012

Sartori, G. (1998). Homo videns. La sociedad teledirigida. Madrid: Taurus.

Sunstein, C. R. (2003). República.com. Internet, democracia y libertad. Paidós: Barcelona.

Tezanos, J. F. (2002). La democracia incompleta: el futuro de la democracia postliberal. Biblioteca Nueva: Madrid.

Thompson, J. (1998). Los media y la modernidad. Barcelona: Paidós.

Thelwallm, M.; Buckley, K. y Paltoglou, G. (2011) . "Sentiment in Twitter Events". En Journal of the American Society for Information Science and Technology, $\mathrm{n}^{\circ} 62,406-418$. DOI: 10.1002/asi.21462.

Tumasjan, A.; Sprenger, T.; Sandner, P. y Welpe, I. (2011). "Predicting elections with Twitter: what 140 characters reveal about political sentiment". En Proceedings of the Fourth International Conference on Weblogs and Social Media (178-185), febrero. Washington: AAAI Press.

Zappavigna, M. (2012). The discourse of Twitter and social media. London: Continuum. 
